\title{
Roles of the medial prefrontal cortex, mediodorsal thalamus, and their combined circuit for performance of the odor span task in rats: analysis of memory capacity and foraging behavior
}

\author{
Gavin A. Scott, Max C. Liu, Nimra B. Tahir, Nadine K. Zabder, Yuanyi Song, \\ Quentin Greba, and John G. Howland \\ Department of Anatomy, Physiology, and Pharmacology, University of Saskatchewan, Saskatoon, SK, S7N 5E5, Canada
}

\begin{abstract}
Working memory (WM), the capacity for short-term storage of small quantities of information for immediate use, is thought to depend on activity within the prefrontal cortex. Recent evidence indicates that the prefrontal neuronal activity supporting WM is driven by thalamocortical connections arising in mediodorsal thalamus (mdThal). However, the role of these connections has not been studied using olfactory stimuli leaving open the question of whether this circuit extends to all sensory modalities. Additionally, manipulations of the mdThal in olfactory memory tasks have yielded mixed results. In the present experiment, we investigated the role of connections between the rat medial prefrontal cortex (mPFC) and mdThal in the odor span task (OST) using a pharmacological contralateral disconnection technique. Inactivation of either the MPFC or mdThal alone both significantly impaired memory performance in the OST, replicating previous findings with the mPFC and confirming that the mdThal plays an essential role in intact OST performance. Contralateral disconnection of the two structures impaired OST performance in support of the idea that the OST relies on mPFC-mdThal connections, but ipsilateral control infusions also impaired performance, complicating this interpretation. We also performed a detailed analysis of rats' errors and foraging behavior and found a dissociation between $\mathrm{mPFC}$ and mdThal inactivation conditions. Inactivation of the mdThal and mPFC caused a significant reduction in the number of approaches rats made per odor, whereas only mdThal inactivation or mPFC-mdThal disconnection caused significant increases in choice latency. Our results confirm that the mdThal is necessary for performance of the OST and that it may critically interact with the mPFC to mediate OST performance. Additionally, we have provided evidence that the mPFC and mdThal play dissociable roles in mediating foraging behavior.
\end{abstract}

Working memory (WM) is the ability to maintain small amounts of information over a brief delay period for use and manipulation. The prefrontal cortex (PFC), particularly the primate dorsolateral and rodent medial (mPFC) prefrontal cortices, has been shown to play a critical role in maintaining WM information over a delay period (Kamigaki and Dan 2017). The physiological mechanism by which prefrontal neurons maintain stimulus representations over a delay is a contested issue (Stokes 2015; Silvanto 2017; Lundqvist et al. 2018), but a large body of evidence has led to the popular view is that the information held in WM is encoded in the form of prefrontal activity manifested either in persistent firing of individual neurons (Fuster and Alexander 1971) or transient activity of groups of neurons that collectively "tile" the delay period (Constantinidis et al. 2018).

In addition to cortical contributions to WM function, recent work has begun to highlight the critical role played by subcortical structures. The mediodorsal thalamus (mdThal) plays a significant role in learning and memory (Mitchell and Chakraborty 2013; Mitchell 2015; Parnaudeau et al. 2018) and has connections with the PFC that also play an important role in learning and memory (Mitchell 2015; Alcaraz et al. 2016). Deficits in these connections are also present in psychiatric disorders. For instance, functional

Corresponding author: john.howland@usask.ca Article is online at http://www.learnmem.org/cgi/doi/10.1101/lm.050195.119. connectivity between the PFC and thalamus, as well as WM performance (Lee and Park 2005), is reduced in people with schizophrenia (Minzenberg et al. 2009). However, the role of the mdThal in WM has been historically controversial (Wolff et al. 2015) with some studies showing minimal effects of mdThal lesions on WM (Zhang et al. 1998; Alexinsky 2001), only transient impairments (Mumby et al. 1993), and impairments that could have resulted from damage to adjacent thalamic nuclei (Young et al. 1996), demonstrating that the mdThal may be only be selectively involved in certain tasks or non-WM aspects of task performance (Wolff et al. 2015).

Several recent and prominent studies have demonstrated that WM performance as well as persistent activity during WM is supported by thalamocortical loops between the PFC and mdThal (Floresco et al. 1999; Parnaudeau et al. 2013; Bolkan et al. 2017; Bray 2017; Guo et al. 2017; Schmitt et al. 2017). Bolkan et al. (2017) optogenetically inhibited projections from the mdThal to the mPFC of mice during a delayed nonmatch-to-sample (DNMTS) t-maze task and found that spatial WM was impaired when these connections were inhibited

(C) 2020 Scott et al. This article is distributed exclusively by Cold Spring Harbor Laboratory Press for the first 12 months after the full-issue publication date (see http://learnmem.cshlp.org/site/misc/terms.xhtml). After 12 months, it is available under a Creative Commons License (Attribution-NonCommercial 4.0 International), as described at http://creativecommons.org/licenses/by-nc/4.0/. 
specifically during the delay period. Schmitt et al. (2017) optogenetically inhibited mdThal projections to the mPFC during a task in which mice were required to use an auditory cue presented before a delay to use one of two "rules" to select either a visual or auditory stimulus for a reward. Optogenetic inhibition of the mdThal during the delay period impaired performance in addition to dampening rule-selective responses in the PFC. Collectively, these recent studies convincingly show a role for mdThal projections to the $\mathrm{mPFC}$ in maintaining WM representations over a delay. However, no study has yet examined the role of PFC-thalamus connections in an olfactory WM task.

The odor span task (OST) is an incrementing DNMTS task with olfactory stimuli which is used in rodents to measure olfactory WM capacity (Davies et al. 2013a,b; Dudchenko et al. 2000, 2013; Scott et al. 2018). Previous work in our laboratory has shown that performance of the OST relies on the MPFC and dorsomedial striatum (Davies et al. 2017a,b). We have also recently found that delay period spiking of $\mathrm{mPFC}$ neurons is predictive of WM performance in the OST (De Falco et al. 2019), showing an involvement of delay period activity in the mPFC. However, we found in a separate experiment that performance of the OST is independent of the parietal cortex (Scott et al. 2018), in contrast to ample previous research showing an important role for the PC in WM. This result brings into question whether other circuits well known to be involved in WM might be unnecessary for olfactory WM.

Moreover, there remains considerable doubt regarding exactly how the mdThal itself participates in olfactory perception, learning, and memory. The mdThal receives olfactory projections (Powell et al. 1965) and has been referred to as the "olfactory thalamus" (Courtiol and Wilson 2015, 2016), but the precise role of the mdThal in olfactory processing remains unclear (Courtiol and Wilson 2015). Neurons in the mdThal show single-unit activity and increased expression of c-Fos in response to olfactory stimuli (Courtiol and Wilson 2014; Fredericksen et al. 2019) as well as task-related activity during olfactory discrimination (Courtiol and Wilson 2016). However, lesions targeting the mdThal do not cause anosmia in rats (Eichenbaum et al. 1980), and studies assessing the effects of mdThal lesions on odor discrimination have produced mixed findings in which impairments are typically seen in more difficult odor discriminations, are more severe with larger lesions, and manifest as a slower learning curve which can be overcome with intensive training (Eichenbaum et al. 1980; Staubli et al. 1987; Slotnick and Risser 1990). With respect to the role of the mdThal in olfactory learning and memory, lesions of the mdThal impair odor reversal learning (Slotnick and Kaneko 1981; Staubli et al. 1987; Slotnick and Risser 1990; McBride and Slotnick 1997), but produce discrepant results in olfactory DNMTS (Koger and Mair 1994; Zhang et al. 1998).

In light of the discordant literature regarding the role of the mdThal in WM and in olfactory processing, it stands as an open question whether performance of the OST relies on the mdThal-mPFC circuit. In the present experiment, we sought to investigate the role of interactions between the mdThal and the mPFC in rats during the OST. We examined the behavioral effects of mPFC or mdThal inactivation alone, as well as connections between the two structures. In order to test the role of mdThal-mPFC connections, we used a contralateral disconnection technique which has been previously used to investigate the roles of interareal connections while preserving partial function in each targeted structure (Davies et al. 2017a). Additionally, we performed a detailed analysis of rats' errors and foraging behavior in order to further distinguish the types of impairments caused by disruption of each structure. To the best of our knowledge, these types of behavioral analyses have not been reported for the OST to date.

\section{Results}

\section{Cannulae placements}

All rats had infusion sites acceptably within the mPFC and mdThal (Fig. 1). Infusions in the mPFC mainly targeted the prelimbic area. All rats had damage to the cortex overlying the mPFC infusion sites as well as damage to the cortex and dorsal hippocampus overlying the mdThal.

\section{Inactivation of the medial prefrontal cortex Memory performance}

Mean $( \pm$ SEM) Span and Best Trial for mPFC inactivations are shown in Figure 2A. A repeated measures one-way ANOVA conducted on Mean Span between Sham performance, Saline, and $\mathrm{M} / \mathrm{B}$ revealed a significant main effect of Drug treatment $\left(F_{(2,56)}=\right.$ 35.44, $P<0.0001)$. Tukey-corrected multiple comparisons revealed that Mean Span was significantly lower in the M/B condition than in the Sham condition $(P<0.0001)$ and the Saline condition $(P<$ 0.01). Interestingly, saline-treated rats were significantly worse than the Sham condition $(P<0.005)$. A repeated measures one-way ANOVA conducted on Best Trial between Sham performance, Saline, and $\mathrm{M} / \mathrm{B}$ revealed a significant main effect of Drug treatment $\left(F_{(2,56)}=33.83, P<0.0001\right)$. Tukey-corrected multiple comparisons revealed that Mean Span was significantly lower in the

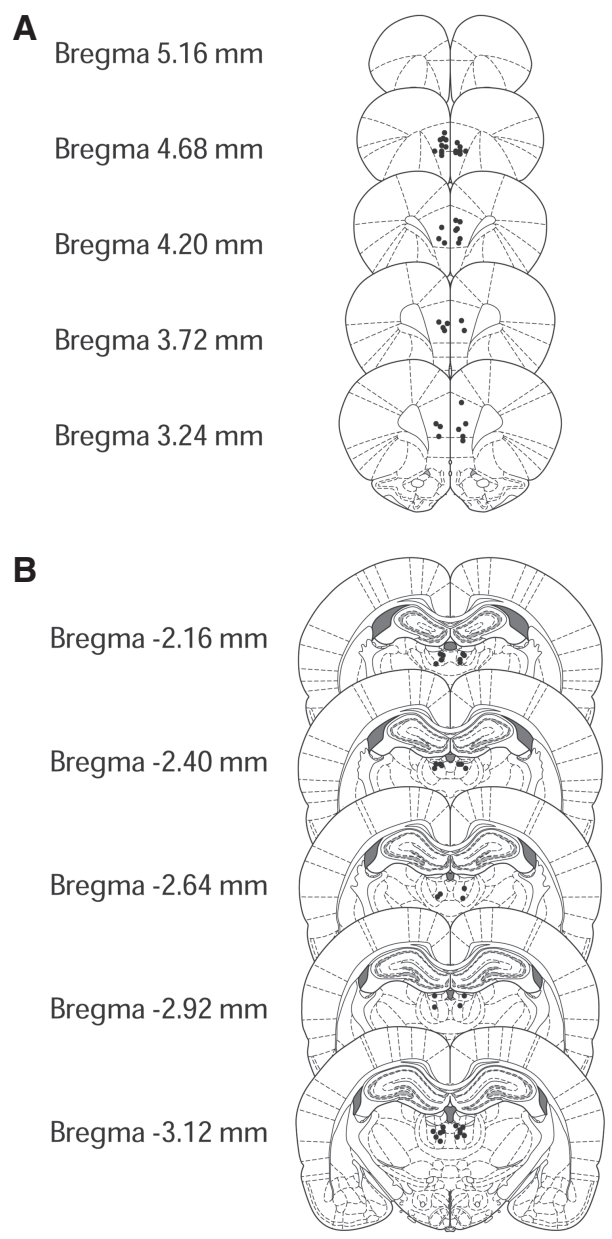

Figure 1. (A) Approximate locations of infusions into the mPFC. (B) Approximate locations of infusions into the mdThal. Illustrations were adapted from Paxinos and Watson (2006), with permission from Elsevier 2019. 
A
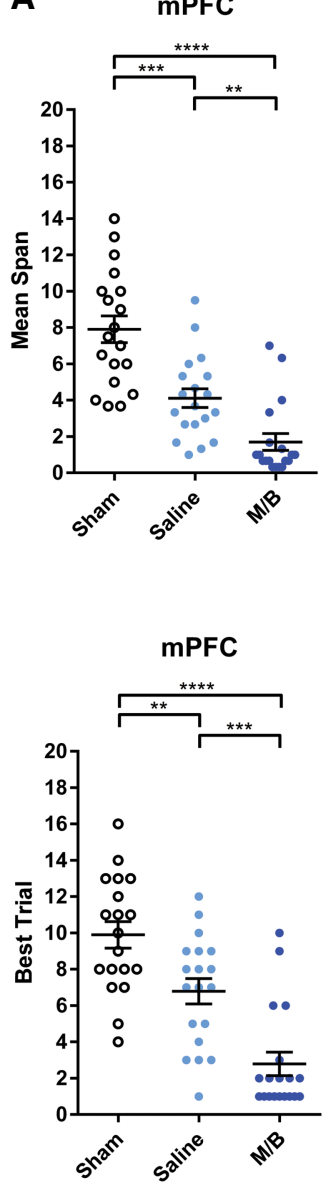

B
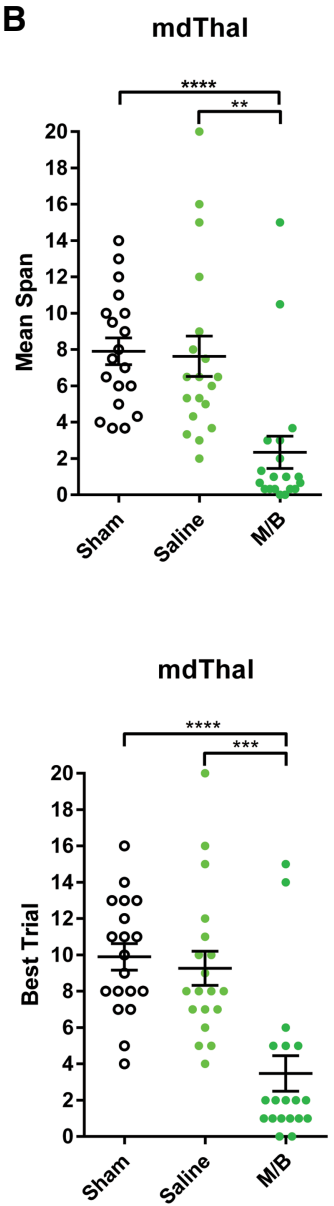

C

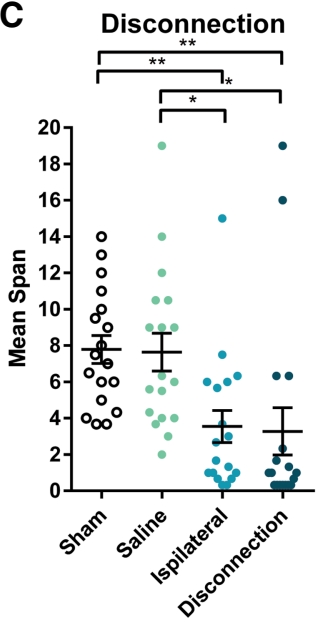

Disconnection

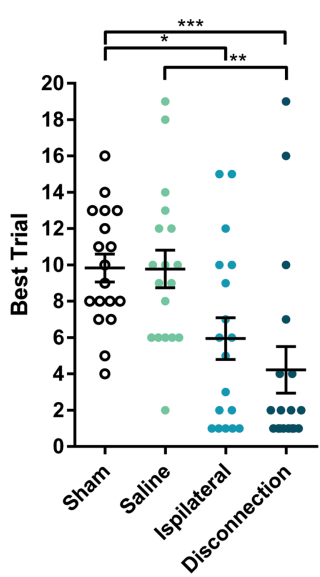

Figure 2. (A) Mean ( \pm SEM) Span and Best Trial after Sham, Saline, and after $M / B$ infusions into the mPFC. Infusions of M/B into the MPFC significantly impaired Mean Span and Best Trial, replicating previous findings that the MPFC is critical for OST performance. Interestingly, Saline infusions also caused a significant impairment relative to Sham infusions, demonstrating that the Saline control is not, itself, entirely inert; $(B)$ Mean $( \pm S E M)$ Span and Best Trial after Sham, Saline, and M/B infusions into the mdThal. Infusions of $M / B$ into the mdThal significantly reduced both Mean Span and Best Trial, demonstrating that the mdThal is critical for intact OST performance. (C) Mean ( \pm SEM) Span and Best Trial after Saline infusions, Ipsilateral M/B infusions, or disconnection of the mPFC and mdThal. Disconnection caused a significant impairment in both Mean Span and Best Trial, whereas Ipsilateral infusions caused a significant impairment only in Mean Span relative to Saline, although both Mean Span and Best Trial were impaired relative to Sham infusions. The results are not inconsistent with the interpretation that mPFC-mdThal connections are necessary for the OST, but the impairment in the Ipsilateral control condition means other explanations cannot be ruled out.

$\mathrm{M} / \mathrm{B}$ condition than in the Sham condition $(P<0.0001)$ and the Saline condition $(P<0.001)$. Best Trial in the Saline condition was also significantly lower than in the Sham condition $(P<0.01)$.

\section{Error position and foraging effects}

Mean ( \pm SEM) Choice Latency, mean error position (mEP), percentage of total odor approaches to the error odor (\%AppE), and average approaches per odor (App/O) after Saline or M/B infusions into the mPFC are shown in Figure 3. A dependent $t$-test revealed no significant difference in Choice Latency between Saline and M/B infusions $\left(t_{(18)}=1.1, P=0.29\right)$. After filtering by exclusion criteria for our error analysis, the sample size was $n=18$ for Saline and $n=10$ for $\mathrm{M} / \mathrm{B}$ for the $\mathrm{mEP}$ and App/O analyses and, for the \%AppE analysis, $n=17$ for Saline and $n=10$ for M/B. Independent $t$-tests revealed no statistically significant differences between Saline and
$\mathrm{M} / \mathrm{B}$ on $\mathrm{mEP}\left(t_{(26)}=0.59, P=0.56\right)$ or $\% \mathrm{AppE}$ $\left(t_{(25)}=1.66, P=0.11\right)$. In contrast, mean App/O was significantly lower in the $\mathrm{M} / \mathrm{B}$ condition than the Saline condition $\left(t_{(26)}=3.06, P<.01\right)$, indicating that in the $\mathrm{M} / \mathrm{B}$ condition, rats made significantly fewer approaches to each odor.

\section{Inactivation of the mediodorsal thalamus}

\section{Memory performance}

Mean $( \pm$ SEM) Span and Best Trial are shown in Figure $2 \mathrm{~B}$. A repeated measures one-way ANOVA revealed a significant main effect of Drug treatment $\left(F_{(2,56)}=13.92, P<0.0005\right)$. Tukey-corrected multiple comparisons revealed that Mean Span was significantly lower in the $\mathrm{M} / \mathrm{B}$ condition than in the Saline $(P<0.005)$ or Sham $(P<$ $0.0001)$ conditions and the Saline and Sham conditions were not significantly different $(P=0.98)$. A one-way repeated measures ANOVA revealed a main effect for Best Trial $\left(F_{(2,56)}=18.98, P<\right.$ $0.0001)$. Tukey post hoc tests revealed that rats' Best Trial in the M/B condition was significantly lower than both the Saline condition $(P<0.001)$ and the Sham Infusion condition $(P<0.0001)$ while there was no difference between the Saline and Sham Infusion conditions $(P<0.84)$.

\section{Error position and foraging effects}

Mean ( \pm SEM) Choice Latency, mEP, \%AppE, and App/O for mdThal inactivations are shown in Figure 4. A dependent $t$-test on revealed that rats had significantly higher Choice Latency in the $\mathrm{M} / \mathrm{B}$ condition than the Saline condition $\left(t_{(18)}=3.04, P<0.01\right)$. Due to this significant increase in Choice Latency, we sought to determine whether the impaired Mean Span may be related to the increased latency. The relationship between Mean Span and Choice Latency is shown in Figure 5A. Due to the fact that the distribution of choice latencies following mdThal inactivation violated normality $(K 2=7.51, P<0.05)$, a Spearman correlation was conducted and revealed that Mean Span was significantly negatively correlated with Choice Latency $\left(r_{\mathrm{s}}=\right.$ $-0.57, P<0.05)$. After applying exclusion criteria to the error analysis, the sample size was $n=17$ for Saline and $n=11$ for M/B for the mEP analysis, Saline $=14$ and $\mathrm{M} / \mathrm{B}=11$ for the $\% A p p E$ analysis, and Saline $=19$ and $\mathrm{M} / \mathrm{B}=11$ for the App/O analysis. Independent $t$-tests revealed no significant change in $\mathrm{mEP}$ $\left(t_{(26)}=1.86, P=0.07\right)$ or $\% A p p E\left(t_{(23)}=1.74, P=0.09\right)$. The distribution of App/O following mdThal Saline infusions violated normality $(K 2=8.94, P<0.05)$, so a Mann-Whitney test was performed to compare the Saline and $\mathrm{M} / \mathrm{B}$ conditions and revealed that App/O was significantly lower in the $\mathrm{M} / \mathrm{B}$ condition $(U=55, P<0.05)$, indicating the rats made significantly fewer approaches per odor in the $\mathrm{M} / \mathrm{B}$ condition.

\section{Contralateral disconnection of the medial prefrontal cortex and mediodorsal thalamus}

Memory performance

Mean $( \pm$ SEM) Span and Best Trial for mPFC-mdThal disconnection is shown in Figure 2C. One rat which had been included in the 
A Choice Latency

mEP

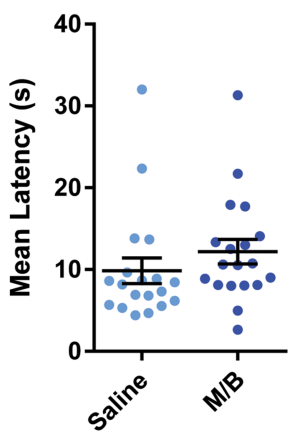

C

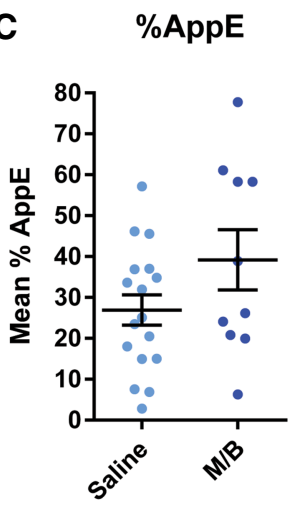

B

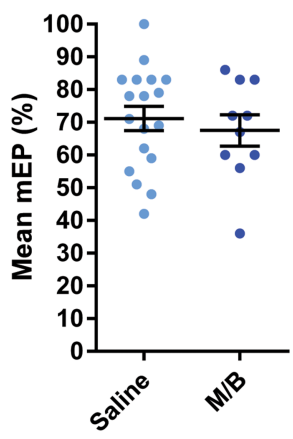

D

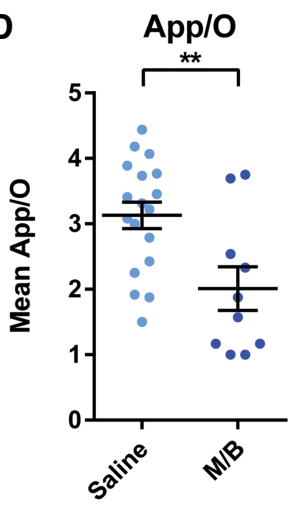

Figure 3. (A) Mean ( \pm SEM) Choice Latency following infusions of Saline or M/B into the mPFC. Rats' Choice Latency was unaffected by $\mathrm{mPFC}$ inactivation. (B) Mean ( \pm SEM) mEP following infusions of Saline or M/B into the MPFC. There was no shift in the mean error position caused by mPFC inactivations. (C) Mean ( \pm SEM) \%AppE following infusions of Saline or M/B into the MPFC. Inactivation of the MPFC did not cause a statistically significant change in the percentage of total odor approaches accounted for by the error odor. (D) Mean ( \pm SEM) App/O following infusions of Saline or M/B into the mPFC. Inactivation of the mPFC caused rats to make significantly fewer approaches per presented odor.

previous analyses was excluded from analyses of memory performance and foraging due to a lack of responding during OST testing following disconnection infusions. A repeated measures one-way ANOVA conducted on Mean Span revealed a significant main effect of Drug treatment $\left(F_{(3,71)}=9.26, P<0.001\right)$. Tukey-corrected multiple comparisons revealed that Mean Span was significantly lower in the Disconnection condition than in the Sham Infusion condition $(P<0.01)$ and the Saline condition $(P<0.05)$. The Sham Infusion and Saline conditions were not significantly different $(P=0.99)$. Additionally, the Ipsilateral Control had significantly worse performance than both the Sham Infusion $(P<0.01)$ and Saline $(P<0.05)$ conditions. There was also no significant difference between the Disconnection and Ipsilateral Control conditions $(P=0.99)$. A oneway repeated measures ANOVA conducted on Best Trial revealed a significant main effect for Best Trial $\left(F_{(3,71)}=11.16, P<0.0001\right)$. Tukey post hoc tests revealed that the Disconnection condition was significantly impaired relative to Saline $(P<0.01)$ and Sham Infusion $(P<0.001)$ conditions. The Sham Infusion and Saline conditions were not significantly different $(P>0.99)$. Although Best Trial was significantly lower in the Ipsilateral Control condition than the Sham Infusion condition $(P<0.05)$, it was not significantly lower than the Saline condition $(P=0.07)$ despite a trend toward impairment. Finally, there was no significant difference between the Disconnection and Ipsilateral Control conditions $(P=0.29)$.

\section{Error position and foraging effects}

Mean $( \pm$ SEM) Choice Latency, mEP, \%AppE, and App/O for mPFC-mdThal disconnection are shown in Figure 6 . The distribution of Choice Latency data violated normality in all conditions $\left(K 2 ' s \geq 6.11, P^{\prime} s<0.05\right)$, so a Friedman test was conducted to compare latencies across conditions and produced a Friedman value of $8.44(P<0.05)$, indicating a significant change in Choice Latency. Dunn's multiple comparisons revealed that Choice Latency was only increased in the Disconnection condition relative to the Saline condition $(P<0.05)$ with no differences between Saline and Ipsilateral $(P=0.99)$ or Ipsilateral and Disconnection $(P=$ 0.06). The relationship between Mean Span and Choice Latency for the Disconnection condition is shown in Figure 5B. To determine whether Mean Span was correlated with Choice Latency in the Disconnection condition, a Spearman correlation was conducted and found no significant correlation $\left(r_{s}=-0.19, P=0.45\right)$. After applying exclusion criteria to the error analysis, the sample size was Saline $=18$, Disconnection $=10$, and Ipsilateral $=14$ for the mEP analysis, Saline $=15$, Disconnection $=10$, and Ipsilateral $=12$ for the $\%$ AppE analysis, and Saline $=18$, Disconnection $=10$, and Ipsilateral $=13$ for the App $/ O$ analysis. One-way between subjects ANOVAS revealed no significant main effects for $\mathrm{mEP}\left(F_{(2,41)}=\right.$ $0.46, P=0.64), \% A p p E\left(F_{(2,36)}=2.28, P=0.12\right)$, or App $/ \mathrm{O}\left(F_{(2,40)}=\right.$ $1.47, P=0.24)$.
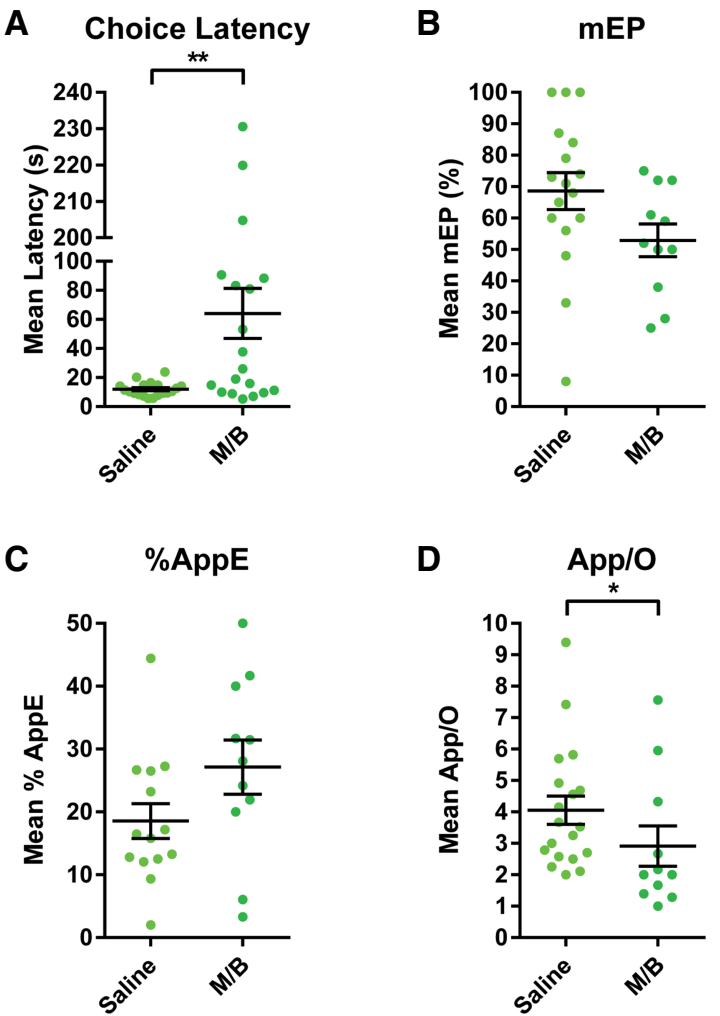

Figure 4. (A) Mean ( \pm SEM) Choice Latency following infusions of Saline or $\mathrm{M} / \mathrm{B}$ into the mdThal. Inactivation of the mdThal caused a large and significant increase in Choice Latency. $(B)$ Mean $( \pm$ SEM) mEP following infusions of Saline or M/B into the mdThal. Inactivation of the mdThal caused no significant change in mean error position. (C) Mean ( \pm SEM) \%AppE following infusions of Saline or $\mathrm{M} / \mathrm{B}$ into the mdThal. Inactivation of the mdThal caused no significant change in the percentage of total approaches accounted for by the error odor. $(D)$ Mean $( \pm S E M)$ App/O following infusions of Saline or $M / B$ into the mdThal. Inactivation of the mdThal caused rats to make significantly fewer approaches per odor. 

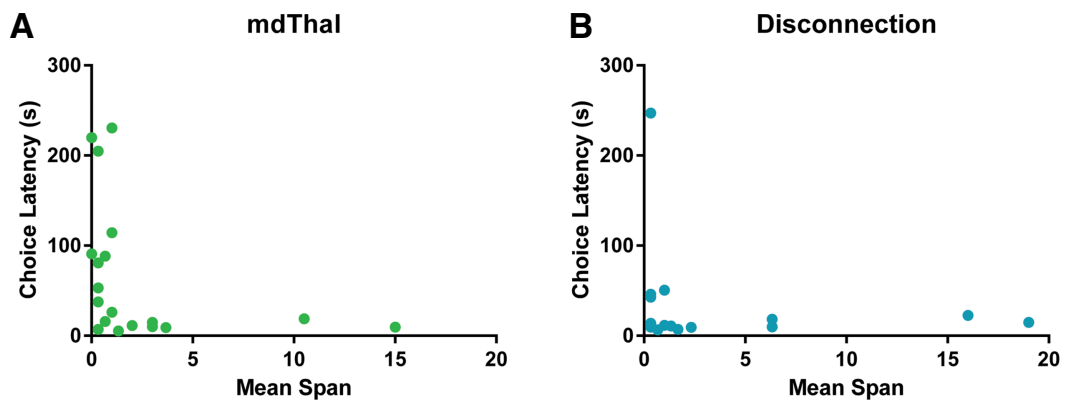

Figure 5. (A) A scatterplot showing the relationship between Mean Span and Choice Latency following mdThal inactivation. Mean Span was significantly negatively correlated with Choice Latency suggesting that the memory impairment following mdThal inactivation may be partly explained by memory decay over increased latencies. (B) A scatterplot showing the relationship between Mean Span and Choice Latency following disconnection of the MPFC and mdThal. Mean Span and Choice Latency were not significantly correlated.

\section{Discussion}

In the present experiment, we found that bilateral inactivation of the mPFC or mdThal both produced severe impairments in memory capacity in the OST. The results replicate previous findings from our laboratory involving mPFC inactivation (Davies et al. 2013a,b). We also provide the first finding, to our knowledge, that the mdThal mediates OST performance, providing more evidence for the uncertain role of the mdThal in WM and olfactory processing (Courtiol and Wilson 2015; Wolff et al. 2015). OST performance was also significantly impaired by contralateral disconnection of the mPFC and mdThal, but ipsilateral control infusions also produced a similar impairment to disconnection. Thus, the present results are more or less in line with previous research demonstrating a role for this circuit in WM (Floresco et al. 1999; Bolkan et al. 2017; Guo et al. 2017; Schmitt et al. 2017), but cannot be conclusively explained as a result of disrupted mPFC-mdThal connections. Additionally, we performed a detailed analysis of rats' foraging behavior and errors and revealed putative differences in the respective roles of the $\mathrm{MPFC}$ and mdThal in mediating rats' search strategies during the OST. Specifically, mPFC and mdThal inactivations both significantly reduced the number of odor approaches rats made before making a choice, while only mdThal inactivation and disconnection of the mdThal from the mPFC caused a significant increase in Choice Latency. Neither contralateral disconnection or ipsilateral control infusions had any significant effects on errors or foraging.

The present results of mPFC inactivation in the OST replicate previous findings from our laboratory (Davies et al. 2013b, 2017a) as well as a large literature demonstrating the critical role of the PFC in WM. Rats' span capacity was significantly reduced following infusions of $\mathrm{M} / \mathrm{B}$, confirming the finding that the $\mathrm{mPFC}$ is critical for OST performance. We have previously demonstrated that the impairment following mPFC inactivation is not due to a loss of olfactory sensitivity (Davies et al. 2013b), meaning that the present results are very unlikely to be the result of a deficit in olfactory perception. Additionally, we saw no change in latency following mPFC inactivation. Our laboratory has found that changes in latency are associated with larger infusion volumes (Davies et al. 2013b) whereas smaller infusion volumes like the one used presently do not increase latency (Davies et al. 2017a). We did not manipulate the delay period in the OST during this experiment, but previous research has produced inconsistent findings with respect to the delay-dependence of mPFC disruption-induced impairments with some authors finding impairments at short/no delays (Joel et al. 1997; Davies
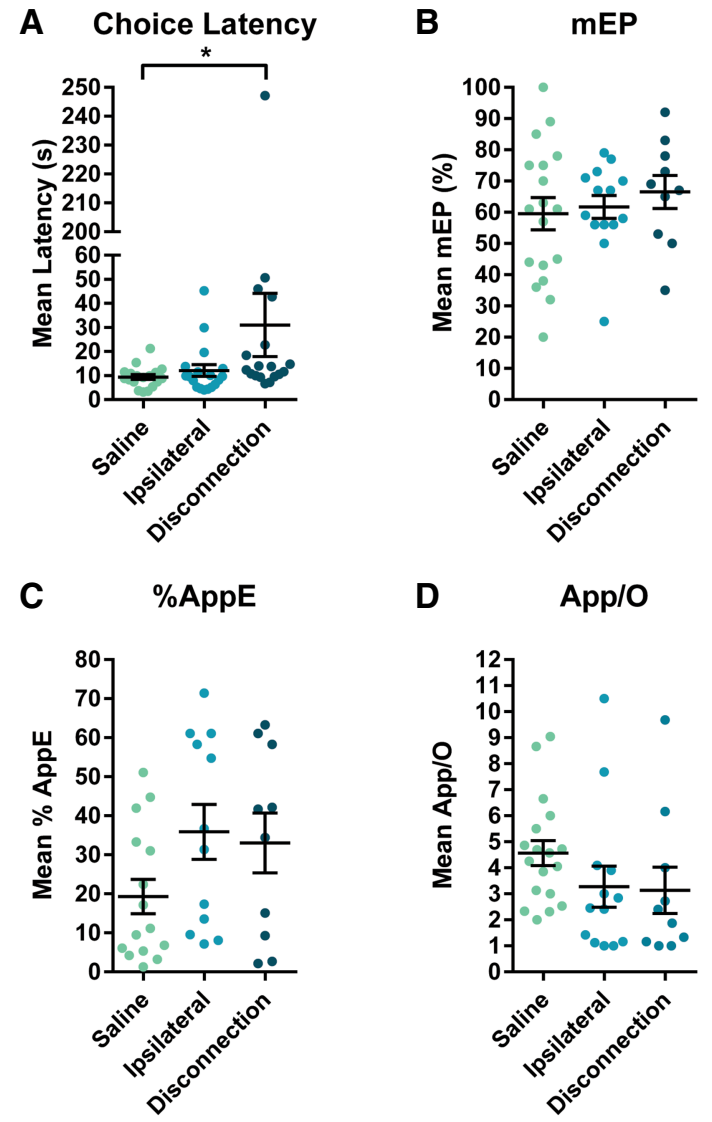

Figure 6. (A) Mean ( \pm SEM) Choice Latency following disconnection of the MPFC and mdThal. Choice Latency was significantly higher in the Disconnection condition than in the Saline condition. $(B)$ Mean $( \pm$ SEM) mEP following disconnection of the MPFC and mdThal. There was no shift in the mean error position caused by disconnection of the two structures. (C) Mean ( \pm SEM) \%AppE following disconnection of the MPFC and mdThal. Disconnection of the two structures did not cause a statistically significant change in the percentage of total odor approaches accounted for by the error odor. $(D)$ Mean ( \pm SEM) App/O following disconnection of the MPFC and mdThal. Disconnection of the two structures caused no significant change in the number of approaches rats made per odor. 
A large literature has previously demonstrated that the mdThal plays a critical role in cognition (Mitchell and Chakraborty 2013; Mair et al. 2015; Mitchell 2015). Although there has been controversy regarding the role of the mdThal in WM with many discrepant results (Wolff et al. 2015). Recent evidence supports the view that it mediates persistent activity in the PFC during WM tasks (Parnaudeau et al. 2013; Schmitt et al. 2017). However, olfactory stimuli have been shown to bypass some circuitry typically associated with WM function such as the posterior parietal cortex (Scott et al. 2018) and hippocampus (Dudchenko et al. 2000), and research on the role of the mdThal in olfactory memory has been produced inconsistent findings (Slotnick and Risser 1990; Koger and Mair 1994; Zhang et al. 1998). The present results confirm that the mdThal plays a role in the OST, contributing to the emerging understanding of the role of the mdThal in olfactory learning and memory tasks (Courtiol and Wilson 2015). Of note, we did not perform any experiments to measure olfactory sensitivity or discrimination, and some previous research has found impaired olfactory discrimination for difficult odor comparisons following mdThal disruption (Eichenbaum et al. 1980; Slotnick and Risser 1990). In the present study, mdThal inactivation did not reduce memory span completely to zero, suggesting that rats at least had sufficient olfactory discrimination abilities to achieve extremely low spans.

Additionally, Choice Latency was significantly increased by both bilateral mdThal inactivation and disconnection. Choice Latency was also negatively correlated with Mean Span in the mdThal inactivation condition. This presents a potential problem in interpreting the impairment in Mean Span given that increased response latency could presumably decrease accuracy by overburdening maintenance processes. While not every rat with a low Mean Span had high Choice Latency following mdThal inactivation, not one rat with high latency obtained a high span. This would suggest that at least some of the impairment in memory performance could be accounted for by memory decay over extended choice latencies. However, it may also be the case that rats achieving higher spans following mdThal inactivation are simply also less impaired in their exploratory foraging behavior and these variables are independent. Previous research has shown that the effects of mdThal disruption on WM tasks can be independent of delay (Mumby et al. 1993; Chauveau et al. 2005).

Despite this potential confound with respect to memory performance, the result points to an interesting role of the mdThal in foraging behavior. Previous research has found that inactivations affecting the mdThal can cause reductions in exploratory motor behavior. For example, Swerdlow et al. (2002) found that inactivation of the mdThal with TTX caused a reduction in motor activity following prepulse inhibition testing, but no reduction in startle magnitude, possibly indicating impaired exploratory motor activity. Additionally, lesions of the mdThal have been shown to attenuate the hyperlocomotor response to apomorphine is rats with 6-hydroxydopamine denervation of the nucleus accumbens (Swerdlow and Koob 1987). This involvement of the mdThal in locomotor behavior is not limited to spontaneous exploration but appears to be involved in goal-directed motor activity as well. An early study by Mogenson and Wu (1988) examined the effects of mdThal inactivation on food hoarding behavior during an open field foraging task and found that mdThal inactivation reduced the number of food pellets hoarded within a $30 \mathrm{~min}$ period. This evidence, combined with the present findings, suggests that a significant component of mdThal involvement in the OST may be in mediating goal-directed foraging behavior.

Importantly, a cause of past controversy over the role of the mdThal in WM has been the fact that the degree of impairment is related to the spread of lesions or infusions to neighboring brain structures with the most specific disruptions sometimes causing minimal impairments (Wolff et al. 2015). Although we did not directly verify the spread of our infusions, our inactivation protocol used similar coordinates and even smaller infusion volumes than what has been previously shown to result in infusate spread confined to the mdThal (Swerdlow et al. 2002). Nonetheless, we cannot rule out the possibility that some adjacent brain structures could have been affected. Furthermore, the role of the mdThal has been additionally controversial due to effects of lesions that are not specific to WM impairments (Wolff et al. 2015). In the present study, we observed significant changes in rats' foraging behavior which could have in turn affected memory performance including a large increase in latency which correlated with memory capacity. Thus, our results should be viewed conservatively as evidence of mdThal involvement in the overall performance of the OST, but the involvement is likely not specific to purely mnemonic processing in the OST.

This is the first study, to our knowledge, to examine the role of mdThal-mPFC connections in an olfactory WM capacity task. Rats had equally impaired Mean Span in the disconnection and ipsilateral control conditions. Although Best Trial for the ipsilateral control was not significantly different than Saline, the trend is clearly toward an impairment nearly as severe as the disconnection. Hence, the present results certainly do not contradict the interpretation that the mPFC-mdThal circuit is necessary for performance of the OST, but the failure of our ipsilateral control leaves open the possibility of several competing interpretations.

It is possible that contralateral projections between mdThal and mPFC play as important a role as ipsilateral connections, unilateral infusions were able to diffuse across the midline to have some effect on the contralateral hemisphere, or that even a unilateral infusion in one or both structures is sufficient to impair performance. The first possibility appears highly unlikely given that tracing studies have found that connections between the two structures appear to be exclusively ipsilateral (Alcaraz et al. 2016). Impairments from interhemispheric diffusion or unilateral inactivation seem unlikely in the case of the mPFC given our lab's previous finding that ipsilateral control infusions which include the mPFC do not significantly impair OST performance (Davies et al. 2017a). Similarly for the mdThal, Floresco et al. (1999) pharmacologically disconnected the mPFC and mdThal and found no impairment in the radial arm maze following unilateral mdThal inactivation at an infusion volume greater than ours. The remaining possibility is that the OST is so sensitive to mdThal disruption that unilateral inactivation possibly including some interhemispheric diffusion is sufficient to impair performance and this would require further experiments to rule out. In any case, the impairments in our disconnection experiment are not conclusively tied to a disruption of mPFC-mdThal connections per se and it is even possible that the two structures are both independently critical for OST performance.

The impairments we observed in OST memory performance may be explained by factors other than deficits in memory capacity per se. Our version of the OST involves a procedure in which odor stimuli are added after each successful choice such that the ratio of familiar odors to the correct novel choice increases in every trial iteration. This attribute has the effect of increasing the number of distractors in addition to increasing memory load such that distractor susceptibility may confound the analysis of memory capacity in addition to underestimating rats' actual memory capacity, a problem that can be overcome by holding constant the number of presented comparison stimuli and computing memory accuracy and distractor susceptibility independently (April et al. 2013). For example, Galizio et al. (2019) 
recently demonstrated that the MK-801 impairment in the OST is partly explained by increased distractor susceptibility. Therefore, it is possible that the present results might similarly be a combined result of impaired memory capacity in addition to increased distractor susceptibility. This interpretation is perhaps supported by our finding that both mPFC and mdThal inactivation caused a reduction in the mean number of approaches per odor which could, in our case, be explained by rats falsely detecting novelty in familiar odors and ending their search. Alternatively, this pattern is consistent with an increase in impulsivity or decrease in behavioral inhibition, an effect previously observed following mPFC disruption (Hardung et al. 2017; Feja and Koch 2014), which could manifest in the OST as a tendency to prematurely choose an odor before searching sufficiently.

As previously mentioned, it is unclear whether the OST impairments caused by mPFC or mdThal inactivation are delaydependent, which may also be problematic for the interpretation that the impairments are specific to mnemonic processes (Wolff et al. 2015). Given the changes in foraging behavior and the possibility that rats were afflicted with increased distractor susceptibility, the present findings may be in line with the interpretation that the mPFC-mdThal circuit is playing an "executive," as opposed to "mnemonic" role consistent with the model of this circuit as an "executive" system (Wolff et al. 2015). Memory capacity for odors may be mediated by the relevant sensory areas, as is suggested by sensory recruitment accounts of WM (Scimeca et al. 2018). It is not known precisely where odor representations in the OST are stored, but candidate structures include primary olfactory cortex such as the piriform (Stettler and Axel 2009) and/or other areas receiving olfactory projections that are relevant to the reward value of stimuli such as the orbitofrontal cortex (Illig 2005) and amygdala (Sosulski et al. 2011).

We attempted to elucidate whether disruption of the mPFC or mdThal affected which odors rats were likely to incorrectly choose when making an error as a function of their position in the sequence of presented odors and, while finding no effect of our manipulations on $\mathrm{mEP}$, we feel this aspect of the OST warrants discussion. Rats are most likely to incorrectly choose odors that are $\sim 70 \%$ of the way through the odor sequence and considerably less likely to incorrectly choose odors occurring nearer the beginning or end of the sequence. It is tempting to make an analogy here to primacy and recency effects present in human WM tasks (Stephane et al. 2010; Botto et al. 2014) and in rats during performance of the radial arm maze (Kesner and Novak 1982; Dimattia and Kesner 1984).

However, our version of the OST allows early-presented odors to be revisited by the rat during each trial iteration violating the serial order of odor presentation. Due to the fact that the most recent odor presented before rats' make an error is only presented in a single trial iteration, it could be argued that the "recency effect" we observe is truly comparable to the recency effect found in humans (Stephane et al. 2010; Botto et al. 2014) and in the serial position curve findings in the radial arm maze (Kesner and Novak 1982; Dimattia and Kesner 1984). In contrast, the "primacy effect" is very likely confounded by repeated rehearsal of familiar odors located early in the sequence. This is evidenced by the prominent skewing of the curve in which the "primacy effect" appears to be of a greater magnitude than the "recency effect." Indeed, previous research controlling when and how often rats are able to revisit familiar odors suggests that the relative familiarity of odors contributes significantly to whether an error is made (April et al. 2013). As such, it is likely inappropriate to make a direct comparison to serial position effects. Nonetheless, we believe this phenomenon in the OST may be of interest to future research.

\section{Conclusions}

The present study examined the relative contributions of the mPFC and mdThal to performance of the OST, an olfactory WM task. Although our laboratory has previously shown that the mPFC is critical for OST performance (Davies et al. 2013b, 2017a), no other study to our knowledge has examined how the mdThal, or its connections with the MPFC, contributes to the OST, and the role of the mdThal in WM and olfactory processing has been controversial (Courtiol and Wilson 2015; Wolff et al. 2015). Inactivation of either the mPFC or the mdThal significantly impaired odor span capacity, as did contralateral disconnection of the two structures. However, ipsilateral control infusions caused an almost equal impairment in memory performance, meaning that impairments in the disconnection may be a result of either interrupted thalamocortical communication or the result of unilateral disruption of one or both structures in an independent fashion. Nonetheless, the results add to previous literature disrupting these connections in other WM tasks and support the view that these connections participate in WM function across sensory modalities. Additionally, these results provide some evidence of dissociations in the effects of mPFC or mdThal inactivation on rats' foraging behavior whereby mdThal inactivations or disconnection cause a reduction in exploratory motor activity in the task similar to previous research on the role of the mdThal in foraging behavior (Mogenson and $\mathrm{Wu} 1988$ ).

\section{Materials and Methods}

\section{Subjects}

Nineteen male Long-Evans rats (Charles River Laboratories) weighing 300-500 g were used. Rats were individually housed in standard ventilated cages and kept on a 12-h light-dark cycle (lights on at 0700). Individual housing was used throughout the experiment to prevent cage mates from tampering with one another's surgical implants and to preclude the possibility of feeding complications such as dominant cage mates depriving submissive cage mates of food. Rats were maintained at $85 \%-90 \%$ of their freefeeding weight with water available ad libitum. All experiments were approved by the University of Saskatchewan Animal Research Ethics Board and conformed to the guidelines of the Canadian Council on Animal Care.

\section{Odor span task}

The OST was performed in a similar fashion to previous experiments from our laboratory (Davies et al. 2013a,b; Murray et al. 2017; Scott et al. 2018) and used a "lid-flipping" procedure adapted from MacQueen et al. (2011). The training was conducted on a table (plywood painted black; $0.84 \mathrm{~m}^{2}$ ) with a $2.5 \mathrm{~cm}$ high border around the edges and elevated $95 \mathrm{~cm}$ above the floor with a metal frame and casters. Between trials, the rats were placed in an opaque Plexiglas enclosure $(32 \mathrm{~cm} \mathrm{~W} \times 50 \mathrm{~cm} \mathrm{H} \times 35 \mathrm{~cm} \mathrm{D})$ located on one side of the table. During trials, the rats were let out of the enclosure and allowed to explore the table. Rewards were presented in plastic Dixie cups $(59 \mathrm{~mL})$ containing a small amount of sand with two reward pellets ( $45 \mathrm{mg}$ rodent purified dustless precision pellets; Bio-Serv) sitting on top of the sand. The portion cups were covered with scented, opaque plastic Dixie cup lids sitting loosely on top of them. The table was lined with 24 holes $(5.5 \mathrm{~cm}$ diameter) drilled into the plywood tabletop along the borders of the open field $3.5 \mathrm{~cm}$ from the table edge and 7.5 $\mathrm{cm}$ from one another. During testing, the portion cups were placed inside of these holes. The lids were scented with odors by storing them in Tupperware containers containing various spices (allspice, anise, basil, cacao, caraway, celery salt, cinnamon, clove, coffee, cumin, dill, fennel, garlic, ginger, lemon, marjoram, mustard, nutmeg, onion, orange, oregano, paprika, sage, and thyme). Lids were replaced each time rats made physical contact 
with them in order to prevent rats from mediating their performance by leaving olfactory cues on the stimuli.

Training consisted of three phases: The first stage was initial shaping, in which rats were habituated to the table and trained to flip lids off the cups to obtain a reward. This was done by first presenting an open cup with a reward and, after each correct trial (the rat successfully obtaining the reward within $5 \mathrm{~min}$ ), an unscented lid was placed on the cup covering incrementally more of the opening (1/2 covered, $3 / 4$ covered, fully covered). This stage continued with each rat receiving three trials per day until they could successfully obtain three rewards in $1 \mathrm{~d}$ from fully covered cups.

The second phase of training was DNMTS training. This stage was conducted by presenting a cup with a sample odor and allowing the rat to obtain the reward. After a delay of $\sim 40 \mathrm{sec}$ (the time between stimulus presentations), a second cup containing a reward with a novel odor (S+) along with an unbaited cup with the original sample odor $(\mathrm{S}-)$ were placed on the table. Importantly, the locations of the novel and familiar odors were shuffled to remove any spatial cues from the task and to prevent rats from visually tracking where the novel odor was placed on the table. Rats were given six trials per day (with an intertrial interval (ITI) of 60

sec) of DNMTS until they performed with $80 \%-100 \%$ accuracy for three out of four consecutive days with the fourth day being no $<50 \%$ accuracy, or until $10 \mathrm{~d}$ of DNMTS training had elapsed, at which point rats were moved to OST training regardless of whether they had reached criterion in DNMTS Rats that were slow learners in DNMTS learned the OST after extensive training even if they had not mastered DNMTS within $10 \mathrm{~d}$.

An illustration of the basic procedure used in the OST is shown in Figure 7A. The OST was performed in the same manner as DNMTS; however, after each correct choice, an additional odor was added to the table in serial fashion until the rat made an error at which point that particular trial was ended and an ITI began before commencing the next trial. As with DNMTS, the locations of the odors were thoroughly shuffled during the $\sim 40 \mathrm{sec}$ delay to remove spatial cues from the task and prevent visually guided mediating strategies. Each rat was trained in this task for a maximum of three trials per day (with no repetition of odors across different trials) or a maximum of $30 \mathrm{~min}$, whichever criterion was met first. Rats were trained for 6-7 wk $\sim 5$ times/wk before being tested with infusions. During training, the span performance, errors, and latency to make a choice were recorded by hand, and latency was tracked manually with a stopwatch. Additionally, video recordings of OST sessions were obtained using a camera located above the arena.

\section{Stereotaxic surgery and intracranial infusions}

Intracranial cannulae implantation as well as the basic infusion procedure were conducted in the same manner described in our previous papers (Davies et al. 2013a, 2017b; Scott et al. 2018). Briefly, rats were implanted with two sets of 23 -gauge stainless steel guide cannulae with one set aimed bilaterally at the mPFC (AP $+3.00 \mathrm{~mm}$; $\mathrm{ML} \pm 0.70 \mathrm{~mm}$; DV $-3.20 \mathrm{~mm}$ ) and another set aimed bilaterally at the mdThal (AP $-2.90 \mathrm{~mm}$; ML $\pm 0.70 \mathrm{~mm}$; DV $-5.00 \mathrm{~mm}$ ). Before infusions were conducted, rats were habituated to the infusion procedure for three consecutive days. Habituation consisted of bringing rats into the infusion room, removing and replacing their dummy stylets, and performing "sham" infusions in which a shortened infusion needle was inserted into the guide can- nulae without piercing the target area and the infusion pump (Harvard Apparatus) was run. On infusion days, rats received infusions of combined muscimol and baclofen $(\mathrm{M} / \mathrm{B}$; each $0.5 \mathrm{mg} / \mathrm{mL}$, mixed together in a 1/1 ratio (Davies et al. 2013b; Scott et al. 2018)) at a rate of $0.3 \mu \mathrm{L} / \mathrm{min}$ to a total infusion volume of $0.3 \mu \mathrm{L}$. The infusions were conducted using 30-gauge stainless steel infusion needles connected to Hamilton syringes with PE50 tubing. The tips of the infusion needles extended $1 \mathrm{~mm}$ past the tips of the guide cannulae when fully inserted. Infusions into the mdThal were performed with $\mathrm{M} / \mathrm{B}$ diluted to half the concentration of that used for the mPFC $(0.25 \mathrm{mg} / \mathrm{mL})$ due to our observation that the $0.5 \mathrm{mg} / \mathrm{mL}$ concentration used in the mdThal frequently resulted in severe locomotor impairments characterized by mild ataxia and hypotonia with a drastic reduction in exploratory behavior. Contralateral disconnections were performed according to the general procedure used in Davies et al. (2017a). M/B was infused unilaterally into contralateral sides of the mPFC and mdThal simultaneously, leaving half of each structure undisturbed, but incapable of communicating with the other structure within hemispheres. The hemisphere (left or right) was counterbalanced between rats. Ipsilateral control infusions were performed by unilaterally infusing $\mathrm{M} / \mathrm{B}$ into ipsilateral sides of the mPFC and mdThal simultaneously in order to control for potential effects of unilateral inactivation.

Infusions were conducted in a semi-counterbalanced fashion over a period of $7 \mathrm{~d}$ in the following order: (1) $2 \mathrm{~d}$ of mdThal infusions in which rats received Saline or M/B in counterbalanced order; (2) $2 \mathrm{~d}$ of mPFC infusions with counterbalanced Saline and M/B conditions; (3) $2 \mathrm{~d}$ of contralateral disconnections in counterbalanced order, and; (4) 1 d of ipsilateral control infusions. This protocol resulted in a total of seven infusions per rat with up to four infusions conducted at any one infusion site. Although a portion of the subjects were administered infusions on consecutive days, we interspersed infusion days with washout days involving normal OST training in a subset of rats and observed no significant loss of performance on the washout days. We have used this general protocol in previous experiments involving pharmacological disconnection (Davies et al. 2017a,b). 


\section{Perfusions and histology}

After the conclusion of behavioral testing, rats were perfused intracardially with either saline $(0.9 \%)$ followed by $30 \%$ formalin or with a solution of formalin, glacial acetic acid, and methanol (FAM). Although two different methods of perfusion were used, we expected there to be no confound caused by the difference in perfusion procedure as we were examining only cannula placement and did not perform any volumetric analyses. Before sectioning, brains were transferred to a cryoprotectant solution of $0.1 \%$ sodium azide and $30 \%$ sucrose and left until they were no longer buoyant. Brains were sectioned coronally at $40 \mu \mathrm{m}$ on a freezing sliding microtome (Leica) and the sections were mounted to glass slides. Assessment of infusion placements was performed by comparing mounted sections to a rat brain atlas (Paxinos and Watson 2006).

\section{Analysis of behavior}

\section{Span capacity}

Memory capacity in the OST was defined as the mean number across trials of odors rats could reach minus the first odor presented and the final odor if the rat made an error on that trial, resulting in a "Mean Span" variable. Additionally, the variable "Best Trial" was used to express span capacity on rats' Best Trial during a given testing session.

\section{Choice Latency}

Choice Latency was measured from the beginning of a trial iteration to when the rat made a choice (flipped a lid off of a cup) and reported as an average across all trial iterations for a given session.

\section{Error position and foraging}

In addition to our analysis of span capacity and latency, we sought to interrogate more subtle effects on rats' exploration of odors, by quantifying the number of times rats "approached" each odor. An "approach" was operationally defined as any instance in which a rat's nose was within $\sim 2 \mathrm{~cm}$ of an odor cup with a heading within $\sim 180^{\circ}$ of the odor cup without displacing the lid and was identified visually by human raters. A rat's nose entering this zone and leaving was counted as a single approach irrespective of how long the rat's nose remained within the zone and a second approach was not counted until the rat's nose left the zone and then subsequently reentered it. We measured several variables related to rats' foraging and errors: (1) As shown in Figure 7B, our particular variant of the OST exhibits a striking "error frequency curve" in which rats under control conditions most often make errors by incorrectly choosing odors that are presented $\sim 60 \%-70 \%$ of the way through the odor sequence. We compared the mean error position (mEP) by standardizing the serial position of "error odors" (the odor that was incorrectly chosen) as a percentage of progress through the full sequence of presented odors. For instance, if a rat made an error during the fourth trial iteration by incorrectly choosing the second odor from the sequence, the serial position of this error would be 50\%; (2) We quantified what percentage of total odor approaches was comprised by approaches to the error odor (\%AppE) as a measurement of how much rats were benefitting from repeated "rehearsal" of familiar odors in the sequence; (3) We quantified the average number of approaches per odor (App/O) in the sequence as a measure of how much rats were revisiting/comparing between odors in the sequence. Sessions in which rats achieved a Best Trial of less than two were excluded from these analyses case-wise because of the extremely restricted variability in serial position on span lengths any shorter than this. Additionally, some sessions contained no errors and thus could not be used for any error analysis but were included in the analysis of App/O. Unfortunately, the location of the error odor was also not clear in a small subset of videos, making these unusable for the \%AppE analysis.

\section{Statistical analysis}

Mean Span and Best Trial were analyzed as one-way repeated measures ANOVAS with sham infusion performance (taken $1 \mathrm{~d}$ prior to beginning of infusions), saline infusion performance, and $\mathrm{M} / \mathrm{B}$ infusion performance. Choice Latency was analyzed using dependent $t$-tests for mPFC and mdThal bilateral inactivations comparing only Saline and M/B conditions, whereas a one-way repeated measures ANOVA was used to analyze the disconnection experiment to compare Saline, Ipsilateral Control, and Disconnection groups. Due to unequal sample sizes in the mEP, \%AppE, and App/O analyses, the Saline and $\mathrm{M} / \mathrm{B}$ conditions were treated as a between-subjects, rather than paired, conditions and were analyzed using independent $t$-tests in the case of mPFC and mdThal inactivations and using one-way between-subjects ANOVAS for the disconnection experiment. The distribution of data within conditions frequently violated assumptions of normality (as determined by D'Agostino-Pearson tests) and homogeneity of variance (as determined by Bartlett's tests), particularly in the inactivation conditions, so applicable nonparametric tests were performed and those results reported in cases where the outcome differed from the parametric tests.

\section{Acknowledgments}

This work was supported by an NSERC Discovery Grant and CIHR Operating Grant to J.G.H. G.A.S. received scholarship funding from NSERC and the University of Saskatchewan. M.C.L. and Y.S. received scholarship funding from NSERC. N.B.T. received a scholarship from the College of Medicine, University of Saskatchewan.

\section{References}

Alcaraz F, Marchand AR, Courtand G, Coutureau E, Wolff M. 2016. Parallel inputs from the mediodorsal thalamus to the prefrontal cortex in the Rat. Eur J Neurosci 44: 1972-1986. doi:10.1111/ejn.13316

Alexinsky T. 2001. Differential effect of thalamic and cortical lesions on memory systems in the rat. Behav Brain Res 122: 175-191. doi:10.1016/ S0166-4328(01)00182-6

April LB, Bruce K, Galizio M. 2013. The magic number 70 (plus or minus 20): variables determining performance in the Rodent Odor Span Task. Learn Motiv 44: 143-158. doi:10.1016/j.lmot.2013.03.001

Bolkan SS, Stujenske JM, Parnaudeau S, Spellman TJ, Rauffenbart C, Abbas AI, Harris AZ, Gordon JA, Kellendonk C. 2017. Thalamic projections sustain prefrontal activity during working memory maintenance. Nat Neurosci 20: 987-996. doi:10.1038/nn.4568

Botto M, Basso D, Ferrari M, Palladino P. 2014. When working memory updating requires updating: analysis of serial position in a running memory task. Acta Psychol (Amst) 148: 123-129. doi:10.1016/j.actpsy .2014.01.012

Bray N. 2017. Working memory: persistence is key. Nat Rev Neurosci 18: 385385. doi:10.1038/nrn.2017.70

Chauveau F, Célérier A, Ognard R, Pierard C, Béracochéa D. 2005. Effects of ibotenic acid lesions of the mediodorsal thalamus on memory: relationship with emotional processes in mice. Behav Brain Res 156: 215-223. doi:10.1016/j.bbr.2004.05.026

Constantinidis C, Funahashi S, Lee D, Murray JD, Qi X-L, Wang M, Arnsten AFT. 2018. Persistent spiking activity underlies working memory. J Neurosci 38: 7020-7028. doi:10.1523/JNEUROSCI.2486-17 .2018

Courtiol E, Wilson DA. 2014. Thalamic olfaction: characterizing odor processing in the mediodorsal thalamus of the rat. J Neurophysiol 111: 1274-1285. doi: $10.1152 /$ jn.00741.2013

Courtiol E, Wilson DA. 2015. The olfactory thalamus: unanswered questions about the role of the mediodorsal thalamic nucleus in olfaction. Front Neural Circuits 9: 1-8. doi:10.3389/fncir.2015.00049

Courtiol E, Wilson DA. 2016. Neural representation of odor-guided behavior in the rat olfactory thalamus. J Neurosci 36: 5946-5960. doi:10.1523/ JNEUROSCI.0533-16.2016

Davies DA, Greba Q, Howland JG. 2013a. GluN2B-containing NMDA receptors and AMPA receptors in medial prefrontal cortex are necessary for odor span in rats. Front Behav Neurosci 7: 1-8. doi:10.3389/fnbeh .2013 .00183

Davies DA, Molder JJ, Greba Q, Howland JG. 2013b. Inactivation of medial prefrontal cortex or acute stress impairs odor span in rats. Learn Mem 20: 665-669. doi:10.1101/lm.032243.113 
Davies DA, Greba Q, Selk JC, Catton JK, Baillie LD, Mulligan SJ, Howland JG. 2017a. Interactions between medial prefrontal cortex and dorsomedial striatum are necessary for odor span capacity in rats: role of GluN2B-containing NMDA receptors. Learn Mem 24: 524-531. doi:10 $.1101 / \mathrm{lm} .045419 .117$

Davies DA, Hurtubise JL, Greba Q, Howland JG. 2017b. Medial prefrontal cortex and dorsomedial striatum are necessary for the trial-unique, delayed nonmatching-to-location (TUNL) task in rats: role of NMDA receptors. Learn Mem 24: 262-267. doi:10.1101/lm.044750.116

De Falco E, An L, Sun N, Roebuck AJ, Greba Q, Christopher C, Howland JG. 2019. The rat medial prefrontal cortex exhibits flexible neural activity states during the performance of an odor span task. eNeuro 6: 1-16. doi:10.1523/ENEURO.0424-18.2019

Dimattia BV, Kesner RP. 1984. Serial position curves in rats: automatic versus effortful information processing. J Exp Psychol 10: 557-563. doi:10 $.1037 / 0097-7403.10 .4 .557$

Dudchenko PA, Wood ER, Eichenbaum H. 2000. Neurotoxic hippocampal lesions have no effect on odor span and little effect on odor recognition memory but produce significant impairments on spatial span, recognition, and alternation. J Neurosci 20: 2964-2977. doi:10.1523/ JNEUROSCI.20-08-02964.2000

Dudchenko PA, Talpos JC, Young JW, Baxter MG. 2013. Animal models of working memory: a review of tasks that might be used in screening drug treatments for the memory impairments found in schizophrenia. Neurosci Biobehav Rev 37: 2111-2124. doi:10.1016/j.neubiorev.2012 .03 .003

Eichenbaum H, Shedlack KJ, Eckmann KW. 1980. Thalamocortical mechanisms in odor-guided behavior. Brain Behav Evol 17: 255-275. doi: $10.1159 / 000121803$

Feja M, Koch M. 2014. Ventral medial prefrontal cortex inactivation impairs impulse control but does not affect delay-discounting in rats. Behav Brain Res 264: 230-239. doi:10.1016/j.bbr.2014.02.013

Floresco SB, Braaksma DN, Phillips AG. 1999. Thalamic-cortical-striatal circuitry subserves working memory during delayed responding on a radial arm maze. J Neurosci 19: 11061-11071. doi:10.1523/JNEUROSCI .19-24-11061.1999

Fredericksen KE, McQueen KA, Samuelsen CL. 2019. Experience-dependent c-Fos expression in the mediodorsal thalamus varies with chemosensory modality. Chem Senses 44: 41-49. doi:10.1093/chemse/bjy070

Fuster JM, Alexander GE. 1971. Neuron activity related to short-term memory. Science 173: 652-654. doi:10.1126/science.173.3997.652

Galizio M, Deal M, Mathews M, Panoz-Brown D, Prichard A, Bruce KE. 2019. Effects of NMDA antagonist dizocilpine (MK-801) are modulated by the number of distractor stimuli in the rodent odor span task of working memory. Neurobiol Learn Mem 161: 51-56. doi:10.1016/j.nlm.2019 .03 .004

Gulbrandsen TL, Sutherland RJ. 2014. Temporary inactivation of the rodent hippocampus: an evaluation of the current methodology. J Neurosci Methods 225: 120-128. doi:10.1016/j.jneumeth.2014.01.015

Guo ZV, Inagaki HK, Daie K, Druckmann S, Gerfen CR, Svoboda K. 2017. Maintenance of persistent activity in a frontal thalamocortical loop. Nature 545: 181-186. doi:10.1038/nature22324

Hardung S, Epple R, Jäckel Z, Gibor L, Yizhar O, Diester I. 2017. A functional gradient in the rodent prefrontal cortex supports behavioral inhibition. Curr Biol 27: 549-555. doi:10.1016/j.cub.2016.12.052

Illig KR. 2005. Projections from orbitofrontal cortex to anterior piriform cortex in the rat suggest a role in olfactory information processing. J Comp Neurol 488: 224-231. doi:10.1002/cne.20595

Joel D, Weiner I, Feldon J. 1997. Electrolytic lesions of the medial prefrontal cortex in rats disrupt performance on an analog of the Wisconsin Card Sorting Test, but do not disrupt latent inhibition: implications for animal models of schizophrenia. Behav Brain Res 85: 187-201. doi:10 .1016/S0166-4328(97)87583-3

Kamigaki T, Dan Y. 2017. Delay activity of specific prefrontal interneuron subtypes modulates memory-guided behavior. Nat Neurosci 20: 854863. doi: $10.1038 / \mathrm{nn} .4554$

Kesner RP, Novak JM. 1982. Serial position curve in rats: role of the dorsal hippocampus. Science 218: 173-175. doi:10.1126/science.7123228

Koger SM, Mair RG. 1994. A comparison of the effects of frontal cortical and thalamic lesions on measures of olfactory learning and memory in the rat. Behav Brain Res 108: 1088-1100. doi:10.1037/0735-7044.108.6 .1088

Lee J, Park S. 2005. Working memory impairments in schizophrenia: a meta-analysis. J Abnorm Psychol 114: 599-611. doi:10.1037/0021-843X .114 .4 .599

Lomber SG. 1999. The advantages and limitations of permanent or reversible deactivation techniques in the assessment of neural function. J Neurosci Methods 86: 109-117. doi:10.1016/S0165-0270(98)00160-5

Lundqvist M, Herman P, Miller EK. 2018. Working memory: delay activity, yes! persistent activity? maybe not. J Neurosci 38: 7013-7019. doi:10 $.1523 /$ JNEUROSCI.2485-17.2018
MacQueen DA, Bullard L, Galizio M. 2011. Effects of dizocilpine (MK801) on olfactory span in rats. Neurobiol Learn Mem 95: 57-63. doi:10.1016/j.nlm .2010 .11 .004

Mair RG, Miller RLA, Wormwood BA, Francoeur MJ, Onos KD, Gibson BM. 2015. The neurobiology of thalamic amnesia: contributions of medial thalamus and prefrontal cortex to delayed conditional discrimination. Neurosci Biobehav Rev 54: 161-174. doi:10.1016/j.neubiorev.2015.01 .011

McAllister KAL, Saksida LM, Bussey TJ. 2013. Dissociation between memory retention across a delay and pattern separation following medial prefrontal cortex lesions in the touchscreen TUNL task. Neurobiol Learn Mem 101: $120-126$. doi:10.1016/j.nlm.2013.01.010

McBride SA, Slotnick B. 1997. The olfactory thalamocortical system and odor reversal learning examined using an asymmetrical lesion paradigm in rats. Behav Neurosci 111: 1273-1284. doi:10.1037/0735-7044.111 .6 .1273

Minzenberg MJ, Laird AR, Thelen S, Carter CS, Glahn DC. 2009. Meta-analysis of 41 functional neuroimaging studies of executive function in schizophrenia. Arch Gen Psychiatry 66: 811-822. doi:10 .1001 /archgenpsychiatry.2009.91

Mitchell AS. 2015. The mediodorsal thalamus as a higher order thalamic relay nucleus important for learning and decision-making. Neurosci Biobehav Rev 54: 76-88. doi:10.1016/j.neubiorev.2015.03.001

Mitchell AS, Chakraborty S. 2013. What does the mediodorsal thalamus do? Front Syst Neurosci 7: 1-19. doi:10.3389/fnsys.2013.00037

Mogenson GJ, Wu M. 1988. Disruption of food hoarding by injections of procaine into mediodorsal thalamus, GABA into subpallidal region and haloperidol into accumbens. Brain Res Bull 20: 247-251. doi:10.1016/ 0361-9230(88)90185-2

Mumby DG, Pinel JPJ, Dastur FN. 1993. Mediodorsal thalamic lesions and object recognition in rats. Psychobiology 21: 27-36.

Murray BG, Davies DA, Molder JJ, Howland JG. 2017. Maternal immune activation during pregnancy in rats impairs working memory capacity of the offspring. Neurobiol Learn Mem 141: 150-156. doi:10.1016/j.nlm 2017.04.005

Parnaudeau S, Neill PO, Bolkan SS, Ward RD, Abbas AI, Roth BL, Balsam PD, Gordon JA, Kellendonk C. 2013. Inhibition of mediodorsal thalamus disrupts thalamofrontal connectivity and cognition. Neuron 77: 11511162. doi:10.1016/j.neuron.2013.01.038

Parnaudeau S, Bolkan SS, Kellendonk C. 2018. The mediodorsal thalamus: an essential partner of the prefrontal cortex for cognition. Biol Psychiatry 83: 648-656. doi:10.1016/j.biopsych.2017.11.008

Paxinos G, Watson C. 2006. The rat brain in stereotaxic coordinates, 6th ed. Academic Press, New York.

Powell TPS, Cowan WM, Raisman G. 1965. The central olfactory connexions. J Anat 99: 791-813.

Schmitt LI, Wimmer RD, Nakajima M, Happ M, Mofakham S, Halassa MM. 2017. Thalamic amplification of cortical connectivity sustains attentional control. Nature 545: 219-223. doi:10.1038/nature22073

Scimeca JM, Kiyonaga A, D'Esposito M. 2018. Reaffirming the sensory recruitment account of working memory. Trends Cogn Sci 22: 190-192. doi:10.1016/j.tics.2017.12.007

Scott GA, Zabder NK, Greba Q, Howland JG. 2018. Performance of the odour span task is not impaired following inactivations of parietal cortex in rats. Behav Brain Res 341: 181-188. doi:10.1016/j.bbr.2017.12.013

Silvanto J. 2017. Working memory maintenance: sustained firing or synaptic mechanisms? Trends Cogn Sci 21: 152-154. doi:10.1016/j.tics .2017 .01 .009

Slotnick BM, Kaneko N. 1981. Role of mediodorsal thalamic nucleus in olfactory discrimination learning in rats. Science 214: 91-92. doi:10 $.1126 /$ science.7280684

Slotnick BM, Risser JM. 1990. Odor memory and odor learning in rats with lesions of the lateral olfactory tract and mediodorsal thalamic nucleus. Brain Res 529: 23-29. doi:10.1016/0006-8993(90)90807-N

Sosulski DL, Bloom ML, Cutforth T, Axel R, Datta SR. 2011. Distinct representations of olfactory information in different cortical centres. Nature 472: 213-216. doi:10.1038/nature09868

Staubli U, Schottler F, Nejat-Bina D. 1987. Role of dorsomedial thalamic nucleus and piriform cortex in processing olfactory information. Behav Brain Res 25: 117-129. doi:10.1016/0166-4328(87)90005-2

Stephane M, Ince NF, Kuskowski M, Leuthold A, Tewfik AH, Nelson K, McClannahan K, Fletcher CR, Tadipatri VA. 2010. Neural oscillations associated with the primacy and recency effects of verbal working memory. Neurosci Lett 473: 172-177. doi:10.1016/j.neulet.2010.02.025

Stettler DD, Axel R. 2009. Representations of odor in the piriform cortex. Neuron 63: 854-864. doi:10.1016/j.neuron.2009.09.005

Stokes MG. 2015. 'Activity-silent' working memory in prefrontal cortex: a dynamic coding framework. Trends Cogn Sci 19: 394-405. doi:10.1016/j .tics.2015.05.004

Swerdlow NR, Koob GF. 1987. Lesions of the dorsomedial nucleus of the thalamus, medial prefrontal cortex and pedunculopontine nucleus: effects on locomotor activity mediated by nucleus accumbens-ventral 
Prefrontal cortex, mediodorsal thalamus, and span

pallidal circuitry. Brain Res 412: 233-243. doi:10.1016/0006-8993(87) 91129-2

Swerdlow NR, Pitcher L, Noh HR, Shoemaker JM. 2002. Startle gating in rats is disrupted by chemical inactivation but not $\mathrm{D} 2$ stimulation of the dorsomedial thalamus. Brain Res 953: 246-254. doi:10.1016/ S0006-8993(02)03298-5

Wolff M, Alcaraz F, Marchand AR, Coutureau E. 2015. Functional heterogeneity of the limbic thalamus: from hippocampal to cortical functions. Neurosci Biobehav Rev 54: 120-130. doi:10.1016/j.neubiorev .2014.11.011

Young HL, Stevens AA, Converse E, Mair RG. 1996. A comparison of temporal decay in place memory tasks in rats (Rattus norvegicus) with lesions affecting thalamus, frontal cortex, or the hippocampal system. Behav Neurosci 110: 1244-1260. doi:10.1037/0735-7044.110.6 .1244

Zhang Y, Burk JA, Glode BM, Mair RG. 1998. Effects of Thalamic and olfactory cortical lesions on continuous olfactory delayed nonmatching-to-sample and olfactory discrimination on rats (Rattus norvegicus). Behav Neurosci 112: 39-53. doi:10.1037/0735-7044 .112 .1 .39

Received June 24, 2019; accepted in revised form October 16, 2019. 


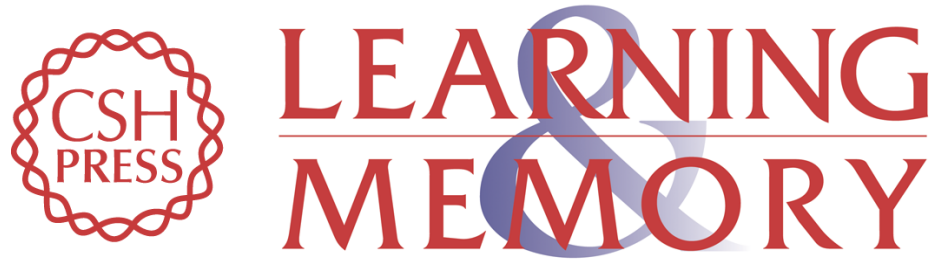

Roles of the medial prefrontal cortex, mediodorsal thalamus, and their combined circuit for performance of the odor span task in rats: analysis of memory capacity and foraging behavior

Gavin A. Scott, Max C. Liu, Nimra B. Tahir, et al.

Learn. Mem. 2020, 27:

Access the most recent version at doi:10.1101//m.050195.119

References This article cites 65 articles, 12 of which can be accessed free at: http://learnmem.cshlp.org/content/27/2/67.full.html\#ref-list-1

Creative This article is distributed exclusively by Cold Spring Harbor Laboratory Press for the Commons first 12 months after the full-issue publication date (see

License http://learnmem.cshlp.org/site/misc/terms.xhtml). After 12 months, it is available under a Creative Commons License (Attribution-NonCommercial 4.0 International), as described at http://creativecommons.org/licenses/by-nc/4.0/.

Email Alerting Receive free email alerts when new articles cite this article - sign up in the box at the Service top right corner of the article or click here. 